\title{
Heart rate variability features for different stress classification
}

\author{
Moridani $\mathrm{MK}^{1}$, Mahabadi $\mathrm{Z}^{2}$, Javadi $\mathrm{N}^{2}$ \\ Department of Biomedical Engineering, Faculty of Health, Tehran Medical Sciences, Islamic Azad University, \\ Tehran, Iran. karimi.m@iautmu.ac.ir
}

\begin{abstract}
AIM: Autonomic nervous system (ANS) activities during different types of stress could affect the electrocardiogram (ECG) signal. This study aimed to recognize the types of stress by using different ECG signals in order to prevent its actual physiological effects on the heart signal.

METHOD: The ECG signal recorded by portable wrist bracelets from 20 students in during seven phases which incorporated three different types of stress and four relaxation phases. After different forms of windowing the signal, we used linear and non-linear features such as detrended fluctuation analysis (DFA), Poincaré plot, approximate and sample entropy, correlation dimension, and recurrence plot to extract various features of the heart rate variability (HRV). Then, different classifiers were used to identify the types of stress. RESULTS: The results showed a decrease in NN50, RMSSD, pNN50, and recurrence plot features, and an increase in the DFA method during stress stages, which show the effect of stress on heart rate. Also, by using the convolutional neural network (CNN), an average classification rate of $98 \%$ was obtained in association with cognitive stress and that of $94.5 \%$ in association with emotional stress.

CONCLUSION: This paper showed that features extracted from HRV can detect the stress and non-stress stages with high significance. Also, the accuracy of this paper proved that the proposed method is successful in preventing the dangerous effects of different types of stress on the heart (Tab. 5, Fig. 6, Ref. 34). Text in PDF www.elis.sk

KEY WORDS: stress, heart rate variability, non-linear features, convolutional neural network, classification.
\end{abstract}

\section{Introduction}

Stress is a nervous tension that has an effect on all functions of the human body. As soon as the brain recognizes the stress, it orders to release neurotransmitters like epinephrine, which leads to the activation of fight-or-flight response. This situation causes physiological disorders because the blood flow in the vital organs such as lung, brain, etc. increases and, consequently, the other internal organs receive a smaller amount of blood (1).

The effects of stress on the body are inflammation, tissue damage, fever, skin allergies, blood pressure, gastrointestinal complications, lack of concentration, cardiovascular disease, diabetes, asthma, etc. (2-3). Moreover, stress affects significantly the heart, which leads to angina, irregular heartbeat, arrhythmia, etc. Chronic stressors can exacerbate diseases as mentioned above, and may even endanger a person's life (4-6).

Stress detection has been assessed by various methods and approaches using ECG and EEG signals, galvanic skin response (GSR), body temperature, and blood volume pulse (7-8). Numerous sensors are used to record these vital signals (9-10). However, when producing such a sensor, it must be taken into consideration

1,2 Department of Biomedical Engineering, Faculty of Health, Tehran Medical Sciences, Islamic Azad University, Tehran, Iran

Address for correspondence: M.K. Moridani, No. 29, Floor 3, Farjam St., Tehran-Pars, Tehran, Iran, Postal Code: 1653989618

Fax: +982188675452 that it should be used during day-to-day activities of patients when they are exposed to normal daily stressors without disturbing their daily rhythm (11). Nowadays, due to the difficulty associated with recording the brain signals, appearance problems, inability to record during daily activities, as well as with invasiveness of the procedure, the study of heart signals is in the center of researchers' attention (12-13).

The ECG signal is analyzed from both short-term and long-term aspects. The long-term reviews provide enough information for detecting ECG signals but the signal-recording process is a bit difficult. Short-term reviews are much easier to record because of less time and smaller volume needed. Many features can be extracted within 20 seconds of a heart rate signal. However, the frequencydomain characteristics cannot be considered in short-term reviews because for extracting these features, we need to have time at the minute of a signal (14).

Plenty of researches on stress detection have been done. In the following section, a number of these studies are discussed. The researches on ECG signals show the link between cardiovascular autonomic activity and stress. The ECG signal can be recorded with a portable sensor easily while the patient is exposed to their daily stress. Joel E. Dimsdale et al examined the effect of psychological stress on the human heart. They have presented an overview of the types of stressors typical for the beginning of the 21 st century, and they have concluded that cardiovascular responses to stress are exquisitely coordinated and functional up to a point. If stress and its causes are continuous, they will lead to disturbances in heart 
619-627

function. This stress effect can be modified firstly by cardiologists themselves or by other people trained to prevent heart disorders (15). Jongyoon Choi et al have presented a method for detecting mental stress using heart rate monitors based on the original dynamic modalities and have extended the methods of HRV analysis by Zhong et al in three respects. They concentrated on heart rate monitoring (HRM) instead of ECG monitors and detected sample stress during daily activities rather than using pharmacological blockade as a stressor and suggested a theoretically alternative criterion for mapping various dynamic modes into SNS and PNS influences. The data were provided via a wearable sensor built by them in an effort to develop some features such as those of an open system, modularity, minimal obtrusiveness, power-efficiency, and low-cost. Heart rate measurements were collected from three subjects under four experimental conditions (two mental stimuli and two relaxation inducements) and detected by R-R series. Their approaches have presented a more meaningful clustering of daily events and a better clue to the consumer's daily stress pattern. Consequently, by estimating the autonomic conditions, habits can be identified amd appropriate feedback provided to the consumer interested in adopting healthier behavior (16). Jianbo Gao et al analyzed HRV in patients suffering from the chronic fatigue syndrome (CFS) as compared with healthy controls by two multiscale methods, adaptive fractal analysis (AFA) and scaledependent Lyapunov exponent (SDLD). These three-day studies were performed on 23 subjects with CFS and 41 healthy subjects. They found that the character of the stress test employed during this study naturally makes their HRV data more non-stationary than those measured in resting states. The presented results suggest that AFA and SDLE could be very useful for the analysis of HRV, when measured in both stationary and non-stationary environments (17). Javdad Birjandtalab et al have studied non-EEG signals for evaluating and mapping the neurological status. After collecting the data of 20 persons by non-invasive wrist-worn biosensor, they analyzed them using the T-SNE dimension reduction method and provided differences between various neurological states (18-19). Tania Pereira et al used a new wearable method to analyze time characteristics, frequency domain, and non-linear heart rate signal criterions in the presence of stress. They collected data from 12 healthy volunteers using a trier social stress test (TSST) in a laboratory context while establishing a real-life state to induce stress. The executive test contained 2 periods; the first one was a baseline period (a short period of silence followed by a period of reading a text) and the second period was experimental (oral presentation followed by arithmetic calculation). This study has illustrated an extensive characterization of HRV that led to a selection of the metrics subset, which could evaluate the physiological interaction of stress in a short-time window. In conclusion, rMSSD, AVNN, SDNN, and pNN20 were evaluated as being the most reliable HRV metric in distinguishing stressful from nonstressful situations within a 50-second window (14).

\section{Methods and materials}

\section{Database}

The data recording procedures, including human subjects described in this work were under the supervision of the UTD IRB \#12-29 (Institutional Review Board at the University of Texas at Dallas, Richardson, Texas, USA). The data were collected from 20 students (14 males, 6 females) at an average age of $25 \pm 5$, average height of $170 \pm 7$, and average weight of $66 \pm 13$.

The volunteers were examined during seven phases as follows: five-minute relaxation provided by meditation tools (level 1 ), standing for a minute followed by walking on a treadmill for 2 minutes and jogging on a treadmill for 2 minutes (level 2), second relaxation for 5 minutes (level 3 ), counting backward by sevens, beginning with 2,485 for 3 minutes, two-minute Stroop test (naming the color of font of typed names of color rather than reading them - if a volunteer makes a mistake they are warned by a buzzer (level 4), third relaxation for 5 minutes (level 5), after a minute of waiting, the volunteers watched a clip of a horror zombie apocalypse movie The Horde (level 6), and the last relaxation

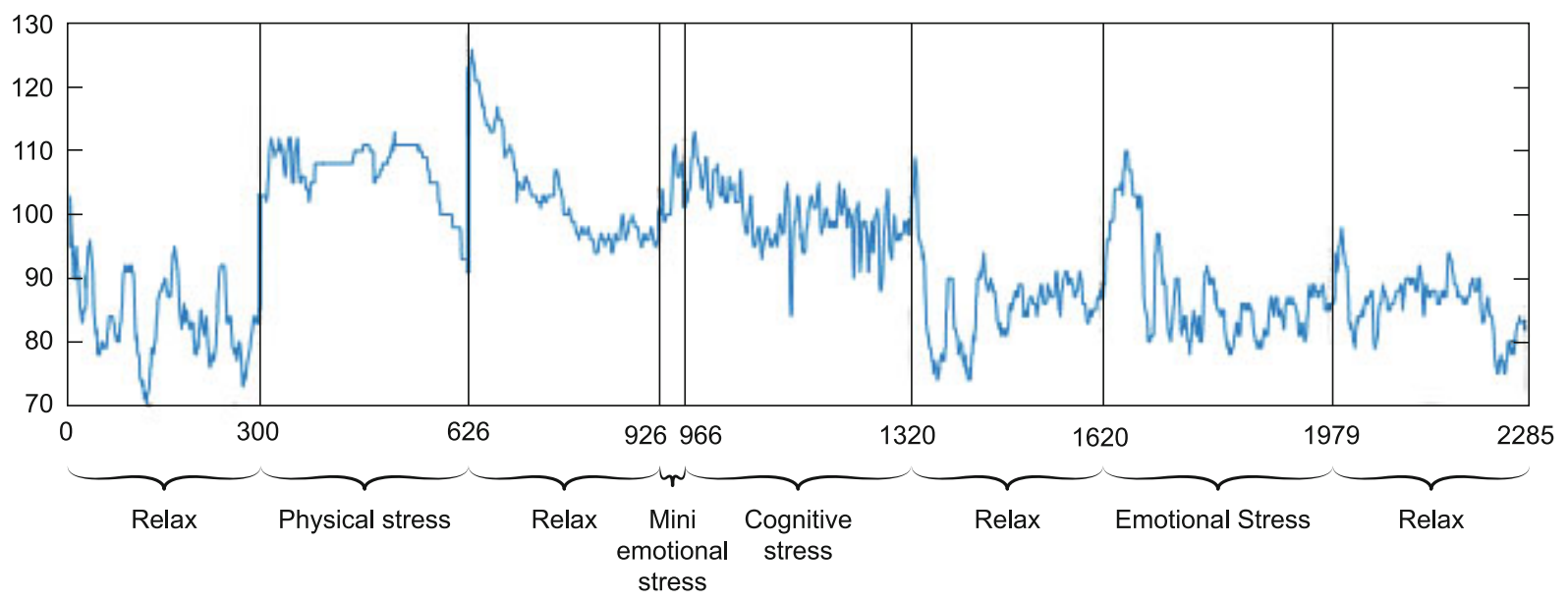

Fig. 1. Heart rate samples from volunteer 16 at different phases. 
for 5 minutes (level 7). Figure 1 shows a sample of various steps that volunteers encountered.

Signals recorded by portable wrist bracelets, which can be used during daily activities. The data were separated into 5-minute windows (except for one that took 40 seconds) evaluated by shorttime analyses.

\section{Proposed algorithm}

A different feature is used in non-linear analysis filed to distinguish between various conditions that volunteers encountered. In the following section, we discuss the introduction of these features.

\section{Detrended fluctuation analysis (DFA)}

DFA is used in the analysis of signals to determine short-term and long-term correlations in a time series of a specific signal. The procedure contains a limited-time series $\left(x_{t}\right)$ of length $\mathrm{N}$ where $t \in N\left(x_{t}\right.$ is the RR distance). Integration or summation converts this phase into an unbounded process $X_{t}(20)$.

\section{Poincaré plot}

The Poincaré plot is a technique derived from non-linear dynamics, and maps the nature of RR intervals fluctuation. In the Poincaré graphs, also referred to as return maps, each RR interval is plotted as a function of the previous RR interval. Thus, the RR time series are plotted in the space of the phase. We consider the point plotted by Poincaré as an ellipse and divided it into two parts with a line. The distribution of the point perpendicular to that line is SD1, and the distribution of the point along that line is SD2. SD1 and SD2 (standard deviations of Poincaré plot) are calculated by equations 1 and 2 (21-22).

$\mathrm{SD}^{2}=\frac{1}{2} \operatorname{Var}\left(R R_{\mathrm{n}}-R R_{\mathrm{n}+1}\right)=\frac{1}{2} S D S D^{2}$

$S D 2^{2}=2 S D R R^{2}-\frac{1}{2} S D S D^{2}$

\section{Approximate and sample entropy}

Approximate entropy (ApEn) is a statistical technique used to quantify the complexity of a signal in a time-series. Sample entropy (SampEn) is a modification of approximate entropy used for evaluating the complexity of physiological time-series and diagnosing diseased states. The sample entropy has data length independence and relatively trouble-free implementation over approximate entropy. The comparison of the patterns with themselves has less approximate entropy values, thus the signals are interpreted very regularly. These self-matches are not involved in sample entropy (23-24).

\section{Correlation dimension}

Correlation dimension is a measure of the system's complexity, it provides the number of a necessary independent variable to describe the system's behavior. The linear determination system has a correct correlation dimension, while the correlation dimension of a chaotic system is a fraction. Nonetheless, a haphazard system can have both the correct dimension and fractal dimension (25).

\section{Recurrence plot}

The recurrence plot is a new method in biological signal processing, while the main advantage of this method is in its application to non-stationary signals. The recurrence plot is a non-linear technique in the analysis of the heart rate signal.

In the recurrence plot, two main small-scale structures are observed: 1) the diagonal line structure of length one, which illustrates two trajectories in a one-time unit, was in the mutually neighboring $\varepsilon$; 2) the vertical line structure of length $v$ represents the trajectory that has not changed significantly for a $v$ time unit. To go over visual expression resulted in the recurrence plot, several measures for complexity, which quantify the small-scale structure in the recurrence plot, are proposed in the paper as a recurrence quantification analysis (QRA). These measures are based on the density of recurrence points and diagonal and vertical line structures in the recurrence plot. Equation 3 shows how to calculate the neighborhood of two points.

$\mathrm{R}(i, j)=\left\{\begin{array}{c}1 \text { if }\|\vec{x}(i)-\vec{x}(j)\| \leq \varepsilon \\ 0 \text { otherwise }\end{array}\right\}$

\section{Determinism}

The percentage of recurrence points that form the diagonal lines (with a minimum length $l_{\text {min }}$ ) over the total recurrence points. Equation 4 shows how to calculate determinism:

$\mathrm{DET}=\frac{\sum_{l=l_{\min }}^{N} \operatorname{lp}(l)}{\sum_{l=1}^{N} l p(l)}$

The length of the diagonal lines parameter $\left(l_{\min }\right)$ in equation (4) is set as a minimum because if $l_{\min }=1$, the determinism will be one, and if a very large $l_{\min }$ is selected, the determinism reliability will be reduced.

\section{Averaged diagonal line length}

Averaged diagonal line length is the average time in which two trajectories are close together and are interpreted as the average of prediction time.

$\mathrm{L}=\frac{\sum_{l=l_{\text {min }}}^{N} l p(l)}{\sum_{l=l_{\text {min }}}^{N} P(l)}$

Longest diagonal line

The length of the longest diagonal line:

$\operatorname{Lmax}=\max \left(\left\{l_{i} ; \mathrm{i}=1, \ldots \ldots, N_{l}\right\}\right)$

$N_{l}$ is the number of diagonal lines in the recurrence plot (26).

\section{Classification algorithms}

In this paper, some common classifiers are used in the field of vital signal processing for identifying the different states of 
the person states and detection of stress. These classifiers are described as follows:

\section{Multilayer perceptron}

The artificial neural network is similar to the human brain because both of them involve a large number of processing and intelligent units called neurons or brain cells. The purpose of the artificial neural network's expansion is obtaining the correlation between input and output data. In the multilayer perceptron (MLP), the number of input layer neurons is determined based on the number of considered data features, and the number of output layer neurons is determined based on the number of classes (23).

In this paper, the MLP network has an input layer, hidden layer, and output layer, which contain 5, 3, and 1 neuron, respectively. The tansig function is used as output and input layer neurons conversion function and the mean square error (MSE) is used as an

Tab. 1. The structure and learning parameters for optimal CNN.

\begin{tabular}{lc}
\hline Parameters & Value \\
\hline Learning rate & 0.05 \\
Kernel size & 10 \\
Pooling size & 3 \\
Number of neurons in fully connected layer & 200 \\
Batch size & 256 \\
Iteration per epoch & 256 \\
Stopping the epoch & 100 \\
\hline
\end{tabular}

error criterion. The maximum number of repetitions for training is 10,000 , and the error value to stop training is $0 / 01$.

\section{Support vector machine (SVM)}

The support vector machine (SVM) is recognized as the oldest and most accurate method among popularly known algorithms. Also, the support vector machine is a training method with a controller, and it is used for classification and regression.

The SVM is a binary classification separating two classes, using a linear boundary. In this method, using all the bands, and optimization algorithm, samples forming the class boundaries are obtained. Generally, this algorithm utilizes a non-linear mapping to convert the main data to a higher dimension, and then in this new dimension, a super plate that separates samples of a class from other classes is searched for. This method is nearly as complicated, and its positive characteristic is independent of the number of training samples, has high number of features, while low numbers of samples can work well. The kernel function can be selected from polynomial functions, RBF functions, hyperbolic tangent functions, or other appropriate functions (23).

\section{K-nearest neighbor}

The k-nearest neighbor (KNN) algorithm is one of the most common algorithms for classification. This algorithm is samplebased and classifies based on the k-nearest neighbor and resemblances of the data. In fact, for each new experimental datum, the

Tab. 2. The recurrence plot analysis of HR for all 20 subjects.

\begin{tabular}{|c|c|c|c|c|c|c|c|c|}
\hline & Relaxation & Physical stress & Relaxation & $\begin{array}{l}\text { Mini-emotional } \\
\text { stress }\end{array}$ & Cognitive stress & Relaxtion & Emotional stress & Relaxation \\
\hline $\begin{array}{l}\text { Lmean } \\
\text { (beats) }\end{array}$ & 45.400 & 38.369 & 79.483 & - & 29.153 & 37.040 & 42.129 & 28.841 \\
\hline $\begin{array}{l}\text { Lmax } \\
\text { (beats) }\end{array}$ & 278 & 198 & 247 & - & 245 & 239 & 290 & 243 \\
\hline $\begin{array}{l}\mathrm{REC} \\
(\%) \\
\end{array}$ & 63.571 & 52.736 & 64.232 & - & 51.549 & 50.306 & 61.889 & 56.399 \\
\hline $\begin{array}{l}\mathrm{DET} \\
(\%)\end{array}$ & 99.905 & 99.936 & 99.978 & - & 99.869 & 99.907 & 99.945 & 99.893 \\
\hline ShanEn & 4.2412 & 4.0762 & 4.8909 & - & 3.9390 & 4.1853 & 4.2804 & 3.9932 \\
\hline
\end{tabular}

Tab. 3. Analysis of some features from all 20 subjects.

\begin{tabular}{lcccccccc}
\hline & Relaxation & Physical stress & Relaxation & $\begin{array}{c}\text { Mini-emotional } \\
\text { stress }\end{array}$ & Cognitive stress & Relaxation & Emotional stress & Relaxation \\
\hline SDNN & 5435.8 & 9427.8 & 15982.4 & 4079.0 & 9384.1 & 6692.7 & 5946.7 & 4724.9 \\
\hline Mean HR & 84.476 & 65.355 & 77.776 & 83.325 & 75.152 & 82.599 & 85.191 & 87.345 \\
\hline STD HR (1/min) & 0.05752 & 0.06890 & 0.12705 & 0.03554 & 0.05482 & 0.07180 & 0.06382 & 0.05531 \\
\hline RMSSD & 45504.9 & 1159.03 & 1568.3 & 1250.9 & 1317.3 & 1000.8 & 1000.3 & 1125.1 \\
\hline NN50 & 134 & 85 & 115 & 16 & 152 & 123 & 156 & 139 \\
\hline pNN50 (\%) & 42.574 & 32.306 & 40.784 & 39.809 & 45.821 & 39.847 & 37.436 & 40.066 \\
\hline DFA, alpha1 & 1.7223 & 1.7414 & 1.8565 & - & 1.6919 & 1.6883 & 1.7417 & 1.7265 \\
\hline DFA, alpha2 & 1.1909 & 1.3492 & 1.3692 & - & 1.3661 & 1.3041 & 1.4118 & 1.2492 \\
\hline
\end{tabular}

SDNN - standard deviation of beats to beats; mean HR - an average of heart rate; standard deviation of heart rate; RMSSD - root mean square difference of beats to continuous beats; NN50 - the difference in beat intervals between consecutive bursts greater than 50 seconds; pNN50 - the proportion of the NN50 divided by the total number of beats; DFA - detrended fluctuation analysis 

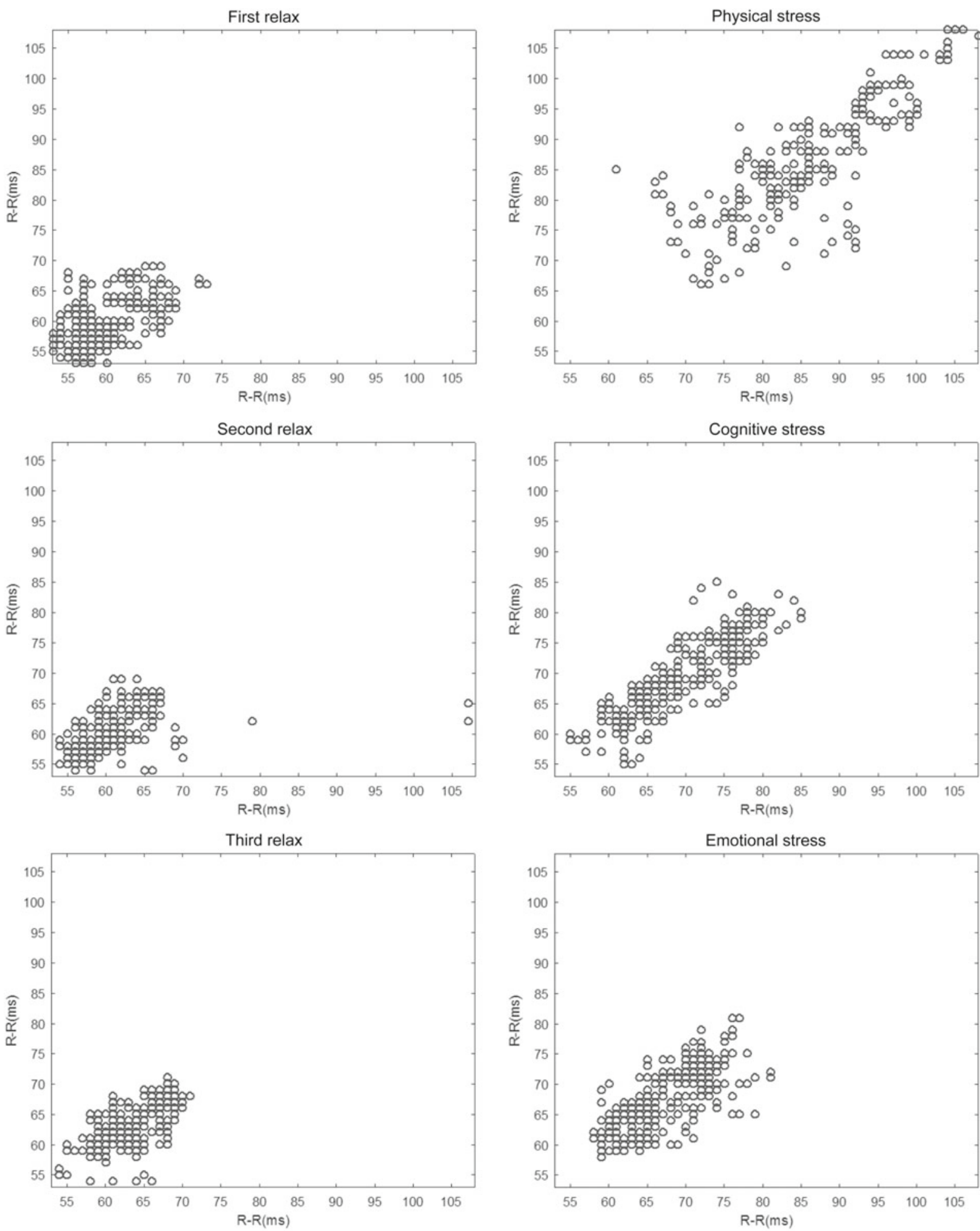

Fig. 2. Poincaré plot of heart signal for volunteer 17 in 6 phases, namely first relaxation, physical stress, second relaxation, cognitive stress, third relaxation, and emotional stress. 


\section{9-627}

k-nearest neighbor distances are calculated and a label similar to the dominant label of k-neighbor for the considered point is determined. The k-nearest neighbor classifier is an algorithm dependent on three factors as follows: $\mathrm{k}$ number neighbor of integer, while changing the value of parameter $\mathrm{k}$ can change the classification result (1), labeled dataset - therefore adding or eliminating any sample to trained samples affects the final decision of the KNN classifier (2), distance criterion which in k-nearest neighbor of Euclidean distance is usually used as a distance criterion to calculate the distance between two samples (3) (27).

\section{Convolutional neural networks (CNN)}

The convolutional neural networks $(\mathrm{CNN})$ indeed describe a specific type of deep learning method. Deep learning is a branch of machine learning discussion and a set of algorithms that attempt to model high-level abstract concepts using training in difficult levels and layers. Deep learning is indeed a new approach to the concept of neural networks that existed for many years, and every few years present a new manner of itself. Determining this point seems necessary that the neural networks have an internal hidden layer, while the network, which has several internal hidden layers, is called a deep network. Deep learning provides us the opportunity to construct a concept with high abstraction using multi-layer learning from down to top (28). Table 1 illustrates the value of $\mathrm{CNN}$ learning parameters introduced in this paper.

\section{Results}

The data were separated into 5-minute windows and analyzed by recurrence plot and parameters such as Lmean, Lmax, REC, DET, and ShanEn as variable time features. These data were also determined at different levels of stress and relaxation and studied afterwards using the mentioned features. These studies are demonstrated in Table 2. As can be seen in this table, there is a difference between stress and relaxation phases, which indicates the effect of stress on autonomic activities and, consequently, on changes in heart rate signals. When the volunteers are in a stressed state, the complexity and mentioned system dimension are changed, which leads to changes in the range of recurrence plot measures, and these changes are evident in Table 2. Therefore, these nonlinear features can be used to analyze the heart rate signal in the presence of stress.

The data which are separated into different widows were analyzed by extracting SDNN, Mean HR, STD HR, RMSSD, NN50, pNN50, and DFA features, and their values are shown in Table 3. The first six of the mentioned characteristics are time-domain features dependent on heart rate. Therefore, if volunteer's heart activities change under stress, these parameters will change too. As can be concluded from Table 3, these parameters changed in different statues, which indicates that the heart rate activity changes in stress and non-stress phases. The last parameter of those seven mentioned features is shown in Table 3. Changes in this parameter in the stress and non-stress phases represent the changes in shortterm and long-term correlations and as a result, they describe the change in the heart activity too.

Figure 2 shows an example of a Poincaré plot in six different levels of stress and non-stress phases (initial relaxation, physical stress, second relaxation, mental stress, third relaxation, and emotional stress) for volunteer number 17 . According to this figure, the accumulation and distribution of points are different in stress and non-stress phases, which illustrates the differences between SD1 and SD2 values and dynamic differences of this signal during the mentioned phases. Figure 2 demonstrates that the density of points in non-stress phases is lower than in stress phases, which means

pNN50

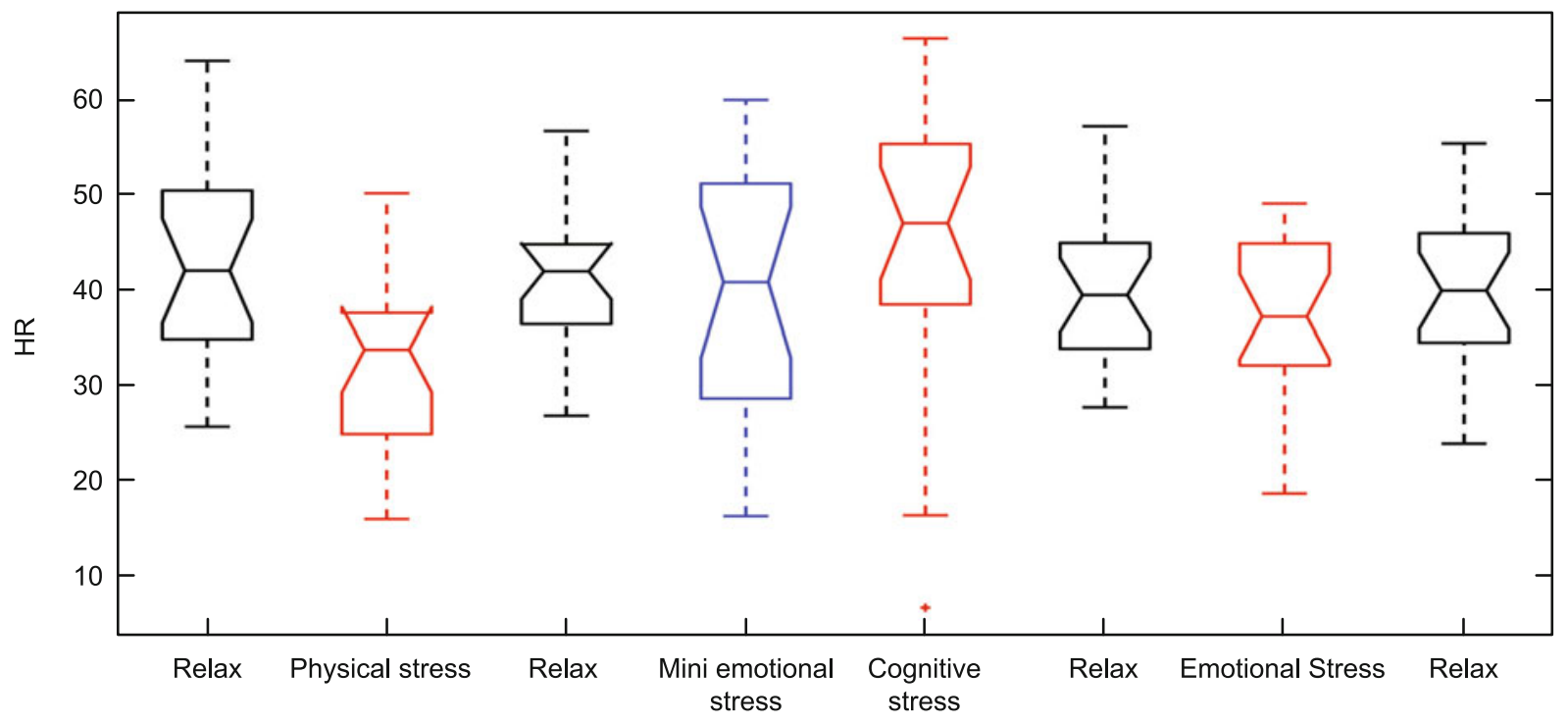

Fig. 3. The boxplot of pNN50 feature in different phases. 
Tab. 4. Average results of different classifiers for recognizing cognitive stress from relaxation.

\begin{tabular}{lcccc}
\hline $\begin{array}{l}\text { Criteria } \\
\text { Classifier }\end{array}$ & $\begin{array}{c}\text { Recall } \\
\%\end{array}$ & $\begin{array}{c}\text { Precision } \\
\%\end{array}$ & $\begin{array}{c}\text { Accuracy } \\
\%\end{array}$ & $\begin{array}{c}\text { F-Score } \\
\%\end{array}$ \\
\hline KNN & 87.0 & 88.8 & 88.0 & 87.9 \\
\hline MLP & 90.0 & 92.8 & 91.0 & 91.4 \\
\hline SVM & 94.0 & 96.9 & 95.5 & 95.4 \\
\hline CNN & 97.0 & 98.9 & 98.0 & 97.9 \\
\hline
\end{tabular}

Tab. 5. Average results of different classifiers for recognizing emotional stress from relaxation.

\begin{tabular}{lcccc}
\hline $\begin{array}{l}\text { Criteria } \\
\text { Classifier }\end{array}$ & $\begin{array}{c}\text { Recall } \\
\%\end{array}$ & $\begin{array}{c}\text { Precision } \\
\%\end{array}$ & $\begin{array}{c}\text { Accuracy } \\
\%\end{array}$ & $\begin{array}{c}\text { F-Score } \\
\%\end{array}$ \\
\hline KNN & 85.0 & 86.7 & 85.5 & 85.8 \\
\hline MLP & 86.0 & 89.6 & 88.0 & 87.8 \\
\hline SVM & 92.0 & 93.9 & 93.0 & 92.9 \\
\hline CNN & 94.0 & 94.9 & 94.5 & 94.4 \\
\hline
\end{tabular}

\begin{tabular}{|c|c|c|}
\hline & \multicolumn{2}{|c|}{ Predicted } \\
\hline \multirow{2}{*}{$\stackrel{\bar{\pi}}{\stackrel{0}{0}}$} & $\begin{array}{c}\text { Correct } \\
\text { True positive }\end{array}$ & $\begin{array}{c}\text { Wrong } \\
\text { False negative }\end{array}$ \\
\hline & $\begin{array}{c}\text { Wrong } \\
\text { False positive }\end{array}$ & $\begin{array}{c}\text { Correct } \\
\text { True negative }\end{array}$ \\
\hline
\end{tabular}
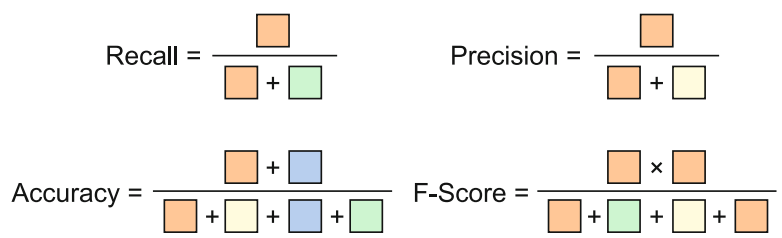

Fig. 4. The efficiency criteria proposed an algorithm. wTP (True Positive): the number of class member and correctly recognized, FP(False Positive): the number of class member and incorrectly recognized, TN (True Negative): the number of a non-member in class and correctly recognized, FN (False Negative): the number of a non-member in class and incorrectly recognized, In this paper, the class member means volunteers in the stress phase.

that the heart dynamic activity is lower in the non-stress phase than in the stress phase. It shows an increase in heart rate in stress phases. The box plot of the pNN50 feature in different phases is shown in Figure 3. The extraction results of these features from the heart rate signal show that the changes in the non-stress phase are approximately equal or smaller than those in the stress phase.

\section{Algorithm assessment}

Firstly, the features introduced in the section before the heart signal of a different person in a different situation were extracted by the proposed algorithm. A proportion of $70 \%$ of the data were used to train the algorithm, and $30 \%$ of them were used for testing. To evaluate the efficiency of the proposed method and introduced classifiers, the confusion matrix was used according to Figure 4

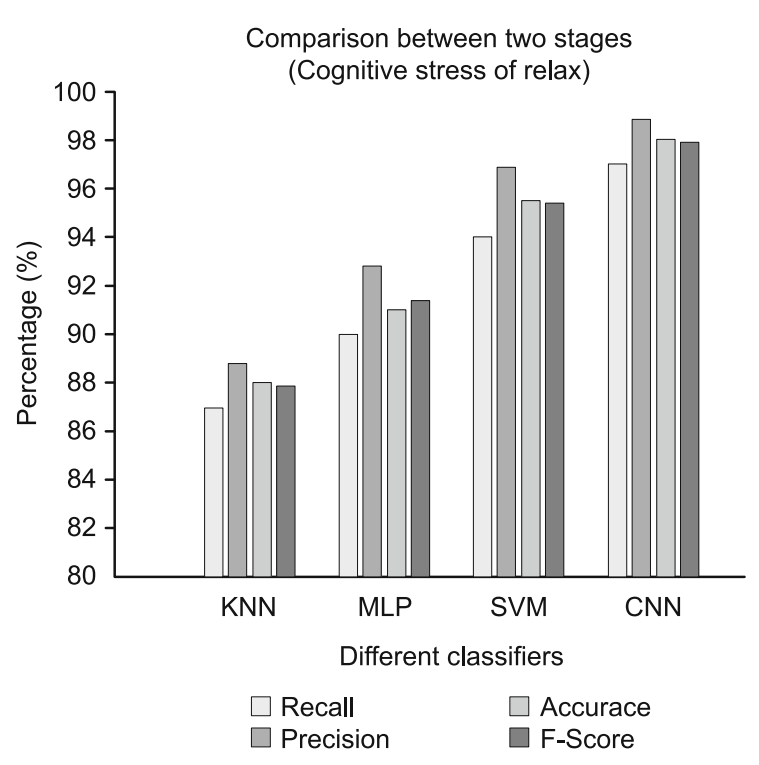

Fig. 5. Different classification functions to detect cognitive stress from relaxation.

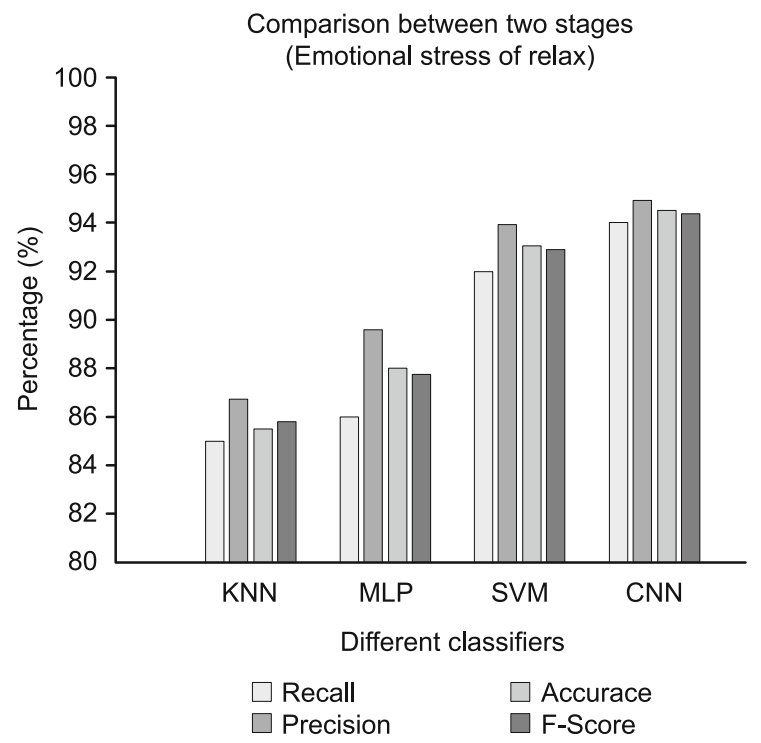

Fig. 6. Different classification functions to detect emotional stress from relaxation.

while the criteria of recall, accuracy, precision, and F-score were introduced.

The proposed algorithms are implemented with MATLAB programming language version 2019.

Tables 4 and 5 show the average results of the proposed algorithm using a 10-fold method and aforementioned various types of classifiers to recognize cognitive stress and emotional stress from the non-stress phase. As can be seen, the usage of deep learning convolutional neural networks provided better results in comparison with other methods. The short training time is one of the 
advantages of this method. Figure 5 shows the efficiency of the proposed algorithm in classifying two phases of cognitive stress and non-stress phase. The precision rate obtained by this classifier is $98.9 \%$ higher compared to other evaluation criteria. Figure 6 also illustrates the efficiency of different classifiers in recognising emotional stress from the non-stress phase. In this case, the value of precision is also greater compared to other criteria.

\section{Discussion}

Stress is one of the parameter disorders of body functions, therefore the ability to recognize it in order to reduce its effects can be very important. Recognizing the stressor factors has been done by verity method, and this task is quite complicated. This study has been done in order to detect stress and alleviate its injurious effect on the heart. Stress can be dangerous for a person with heart disease. Therefore, it should be controlled by analyzing heart rate signals recorded by a comfortably wearable sensor. Also, the use of the heart rate signals to detect stress is easy, straightforward and beneficial for humans. By examining the parameters of heart rate signals, the differences between stress and non-stress phases can be found obviously. The detection of stress from the EEG signal is difficult, therefore its recognition from HRV has been chosen today. Parameters like NN50, RMSSD, PNN50, and recurrence plot features become reduced in the presence of stress while DFA increases.

Mario Salai et al analyzed stress by using a low-cost heart rate sensor and concluded that even a simple, low-cost heart rate monitor device could recognize the characteristics which are subject to change in mental stress. They found that while the mean STDRR, pNN50, RR, and RMSSD in the presence of mental stress decrease, the mean of HR and LF increase remarkably (29). Thoriq Salafi et al has presented a wearable unobtrusive stress-monitoring system using three physiological sensors with a precise algorithm for detecting stress levels, where one of the physiological features for diagnosing the stress was heart rate (30). Alexander Lischke et al found out that people with high HRV have less stress in social life but more stress in family life, at work, and in daily life, which leads to suffering more from stress-related abnormalities as compared with people with low HRV. This study clearly shows the correlation between HRV and stress (31). Also, Karolina Kramaric et al have discovered that the asymmetry of the neonate heart rate is affected by stressful stimuli, which indicates the importance of detecting stress (32).

There are various ways to diagnose stress and control it such as to recognize stress from breathing or saliva but owing to the importance of stress effect on the heart and importance of the heart as the main organ of the human body, the stress detection as measured from the heart rate is more favorable (33-34).

In this paper, the data recorded by a portably wearable sensor were separated into different time windows; afterwards, each window related to stress or non-stress phase was analyzed. The next step illustrates how different types of stress affect heart rate parameters. Our findings are in line with those in other studies in showing that stress has a significant effect on the heart rate sig- nal. A remarkable point in this study lies in the relatively desired results of recognition of the state of stress from non-stress; those results were discussed in detail in the result section.

\section{Conclusion}

The results of this study showed changes in HRV parameters during stress levels by means of different methods and followed and completed some studies which have been done on this topic with greater accuracy. Some studies focused on recognizing stress from other parameters like saliva were investigated too, but this research is about heart rate changes during stress owing to the importance of this topic. The analyzed data clearly illustrate these changes. The variation of recurrence plot and its features like DET, REC, Lmax, Lmean and ShanEn between stress states and non-stress states are visible, and also the time domain features like pNN50, NN50, RMSSD, STD HR, mean HR and DFA are subject to change under stress states too, which was proved by variation of heart rate in different states of stress. The Poincaré plots from different stress states illustrate the dynamic activity of heart rate change during stress too. The box plot of the pNN50 parameter demonstrates this feature in relaxation states as being approximately the same toward stress states and different from each other. Hence stress can be clearly recognized from some features of the heart rate which is a very comfortable non-invasive method that can be used for recording the data during daily activities and thus for detecting the stress encountered by patients, control it, and alleviate its damaging effect on the body as much as possible, especially in those suffering from heart disease.

\section{References}

1. Migra M, Knazeje M, Ochodnicky M, Kovar F, Lacko A, Mokan M. The detection of early myocardial changes in asymptomatic diabetic individuals by $99 \mathrm{mTc}-$ Myoview gated-SPET and heart rate variability measurement. Bratisl Med J 2014; 115 (4); 216-220.

2. Cohena S, Janicki-Devertsa D, Doyleb WJ, Millerc GE, Frankd E, Rabine BS et al. Chronic Stress, Glucocorticoid Receptor Resistance, Inflammation and Disease Risk. PNAS 2012; 109 (16): 5995-5999.

3. Yaribeygi H, Panahi Y, Sahraei H, Johnston TP, Sahebkar A. The impact of stress on body function: A review. EXCLI J 2017; 16: 1057-1072.

4. Zanstraa YJ, Johnston DW. Cardiovascular reactivity in real-life settings: Measurement, mechanisms and meaning. Biol Psychol 2011; 86 (2): 98-105; 1-16.

5. Hamilton JL, Alloy LB. Atypical reactivity of heart rate variability to stress and depression across development: Systematic review of the literature and directions for future research. Clin Psychol Rev 2016; 50; 67-79.

6. Oken BS, Chamine I, Wakeland W. A Systems Approach to Stress, Stressors and Resilience in Humans. Behav Brain Res 2016; 282; 144-145.

7. Van de Vel A, Cuppens K, Bonroy B, Milosevic M, Jansen K, Van Huffel S et al. Non-EEG seizure detection systems and potential SUDEP prevention: State of the art. Seizure 2016; 41; 141-153.

8. de Santos Sierra A, Sánchez Ávila C, Guerra Casanova J, Bailador del Pozo G. A Stress-Detection System Based on Physiological Signals and Fuzzy Logic. Ieee Transact Industr Electronics 2011; 58: 4857-4865. 
9. Rahman MM, Bari R, Ali AA, Sharmin M, Raij A, Hovsepianv K et al. Are We There Yet? Feasibility of Continuous Stress Assessment via Wireless Physiological Sensors. ACM BCB 2014; 2014; 479-488.

10. Goumopoulos C, Menti E. Stress Detection in Seniors Using Biosensors and Psychometric Tests. Procedia Computer Science 2019; 152; 18-27.

11. Salafi T, Kah JCY. Design of Unobtrusive Wearable Mental Stress Monitoring Device Using Physiological Sensor. 7th WACBE World Congress on Bioengineering; IFMBE Proceedings 2015; 52. doi: 10.1007/9783-319-19452-3_4.

12. Cogan D, Birjandtalab J, Nourani M. Multi-Biosignal Analysis for Epileptic Seizure Monitoring. Internat J Neural Systems 2016; 27 (1); 1650031-1650049.

13. Osorio I, Manly BFJ. Probability of detection of clinical seizures using heart rate changes. Seizure 2015; 30; 120-123.

14. Pereira T, Almeida PR, Cunha JPS, Aguiar A. Heart rate variability metrics for fine-grained stress level assessment. Computer Methods Programs Biomed 2017; 148; 71-80.

15. Joel M, Dimsdale E. Psychological Stress and Cardiovascular Disease. J Am Coll Cardiol 2008; 51 (13): 1237-1246.

16. Choi J, Gutierrez-Osuna R. Using Heart Rate Monitors to Detect Mental Stress. Sixth International Workshop on Wearable and Implantable Body Sensor Networks 2009. doi: 10.1109/BSN.2009.13.

17. Gao J, Gurbaxani BM, Hu J, Heilman KJ et al. Multiscale analysis of heart rate variability in non-stationary environments. Front Physiol 2013; 4: 1-8.

18. van der Maaten L, Hinton G. Visualizing Data using t-SNE. J Machine Learning Res 2008; 9; 2579-2605.

19. Birjandtalab J, Cogan D, Baran Pouyan M, Nourani M. A nonEEG Biosignals Dataset for Assessment and Visualization of Neurological Status. IEEE International Workshop on Signal Processing Systems 2016; 110-114. doi:10.1109/SiPS.2016.27.

20. Espen A, Ihlen F. Introduction to multifractal detrended fluctuation analysis in Matlab. Front Physiol 2012; 3; 1-18.

21. Hsu CH, Tsai MY, Huang GS, Lin TC, Chen KP, Ho ST, Shyu LY, Li CY. Poincare plot indexes of heart rate variability detect dynamic autonomic modulation during general anesthesia induction. Acta Anaesthesiol Taiwan 2012; 50 (1): 12-18.
22. Moridani MK, Farhadi H. Heart rate variability as a biomarker for epilepsy seizure prediction. Bratisl Med J 2017; 118 (1): 3-8.

23. Moridani MK, Setarehdan SK, Nasrabadi AM, Hajinasrollah E. Non Linear Feature Extraction from HRV Signal for Mortality Prediction of ICU Cardiovascular Patient. J Med Engineer Technol 2016; 40 (3): 87-98.

24. Montesinos L, Castaldo R, Pecchi L. On the use of approximate entropy and sample entropy with centre of pressure time-series. J NeuroEngineer Rehab 2018; 15 (1): 116-131.

25. Bolea J, Laguna P, Remartínez JM, Rovira E, Navarro A, Bailón R. Methodological Framework for Estimating the Correlation Dimension in HRV Signals. Comput Mathem Methods Med 2014; 129248-129259.

26. Marwan D. Recurence Plots and Cross Recurence Plots. Creative Commons, 2000-2019. [Online]. Available: http://www.recurrence-plot.tk/.

27. Moridani MK, Setarehdan SK, Nasrabadi AM, Hajinasrollah E. A novel approach to mortality prediction of ICU cardiovascular patient based on fuzzy logic method. Biomed Signal Proc Control 2018; 45; 160-173.

28. Raghu S, Sriraam N, Temel Y, Rao SV, Kubben PL. EEG based multi-class seizure type classification using convolutional neural network and transfer learning. Neural Netw 2020; 124: 202-212.

29. Salai M, Vassányi I, Kósa I. Stress Detection Using Low Cost Heart Rate Sensors. Healthcare Engineering 2016; 2016; 5136705-5136718.

30. Salafi T, Kah JCY. Design of Unobtrusive Wearable Mental Stress Monitoring Device Using Physiological Sensor. 7th WACBE World Congress on Bioengineering 2015; 11-14.

31. Lischkea A, Jacksteitb R, Mau-Moellerb A, Pahnkec R, Hamma AO, Weippertc M. Heart rate variability is associated with psychosocial stress in distinct social domains. J Psychosomat Res 2018; 106; 56-61.

32. Kramaric K, Sapina M, Garcin M, Milas K, Piric M, Brdaric D et al. Heart rate asymmetry as a new marker for neonatal stress. Biomed Signal Proc Control 2019; 47; 219-223.

33. Meyera PW, Friederichb HC, Zastrowc A. Breathe to ease - Respiratory biofeedback to improve heart rate variability and coping with stress in obese patients: A pilot study. Mental Health Prevent 2018; 11; 41-46.

34. Machado Fernandez JR, Anishchenko L. Mental stress detection using bioradar respiratory signals. Biomed Signal Proc Control 2018; 43: 244-249. 